\title{
Non-Intersection of Trajectories in Qualitative Phase Space: A Global Constraint for Qualitative Simulationt
}

\author{
Wood W Lee and Benjamin J Kuipers \\ Department of Computer Sciences \\ University of Texas, Austin, Texas 78712
}

\begin{abstract}
The QSIM algorithm is useful for predicting the possible qualitative behaviors of a system, given a qualitative differential equation (QDE) describing its structure and an initial state. Although QSIM is guaranteed to predict all real possibilities, it may also predict spurious behaviors which, if uncontrolled, can lead to an intractably branching tree of behaviors. Prediction of spurious behaviors is due to an interaction between the qualitative level of description and the local state-tostate perspective on the behavior taken by the algorithm.

In this paper, we describe the non-intersection constraint, which embodies the requirement that a trajectory in phase space cannot intersect itself. We develop a criterion for applying it to all second order systems. It eliminates a major source of spurious predictions. Using it with the curvature constraint tightens simulation to the point where system-specific constraints can be applied more effectively. We demonstrate this on damped oscillatory systems with potentially nonlinear monotonic restoring force and damping terms. Its introduction represents significant progress towards tightening QSIM simulation.
\end{abstract}

\section{Introduction}

QSIM [Kuipers, 1986] qualitatively reasons about systems of autonomous qualitative differential equations (QDEs). Although many well known techniques already exist for solving systems of ordinary differential equations (ODEs), they are applicable only to ODEs of restricted forms. In real applications, however, such forms are rare. On one hand, incomplete knowledge often renders QDE models more realistic than exact ODE. On the other hand, even when we do have exact ODEs, they are usually in unsolvable forms. QSIM, always predicting all real solutions to a system of QDEs (in the form of qualitative descriptions of the temporal behavior of parameters), has the potential to deal with these cases.

Taking a phase space view, mathematicians have been able to develop analyses that yield useful global characteristics (such as stability) of solutions to ODEs without explicitly solving them. However, in applications such as

-This work is supported in part by the National Science ndation under grant number IRI-8602665. This paper will vear in the proceedings of AAAI-88. monitoring and control where thresholds are a main concern, such techniques are insufficient. Simulation type techniques, such as QSIM, would be necessary. In such cases, QSIM predictions exhaust all possible manners in which various thresholds might be crossed.

Though a powerful algorithm, a combination of the local state-to-state perspective and the qualitative level of description taken makes it possible for QSIM to predict spurious solutions. In an analysis of the QDE for the damped spring, Lee et al. [1987] identified various new types of constraints (higher derivative, energy and system property) for tightening QSIM simulation. Using early versions of these constraints, they were able to arrive at all and only the correct predictions for the linear damped spring. However, success of these early versions with potentially nonlinear damped springs was not as complete.

Kuipers and Chiu [1987] introduced a generalized higher derivative constraint in the form of curvature constraints. They were able to eliminate a major source of spurious predictions in QSIM, namely, the lack of derivative information, sucessfully. Though a powerful and necessary constraint for simulating systems of second order and higher, there are many cases where curvature constraints alone do not suffice to make predictions tractable.

In this paper, we describe the non-intersection constraint (short for non-intersectionof-phase-space-trajectory constraint). It is not systemspecific in the sense that its derivation does not depend on the specific system QSIM works on. It is derived from a mathematical theorem that governs all systems the current QSIM deals with and applies equally to them. It specifies that phase space trajectories do not cross themselves and eliminates a major source of spurious predictions. We have developed a criterion for applying it to all second order systems. Using it with the curvature constraint tightens simulation to the point where system-specific constraints (such as energy and system property constraints) can be more effectively applied. This is demonstrated on damped oscillatory systems.

In the rest of this paper, we first introduce the phase space framework and how QSIM predictions fit into the picture. Next the non-intersection constraint is described. Then we describe our current implementation and results of applying it to damped oscillatory systems. Its relationship to previously introduced constraints and other issues are discussed. Finally, related work by Sacks [1987] and Struss [1987] are described. 

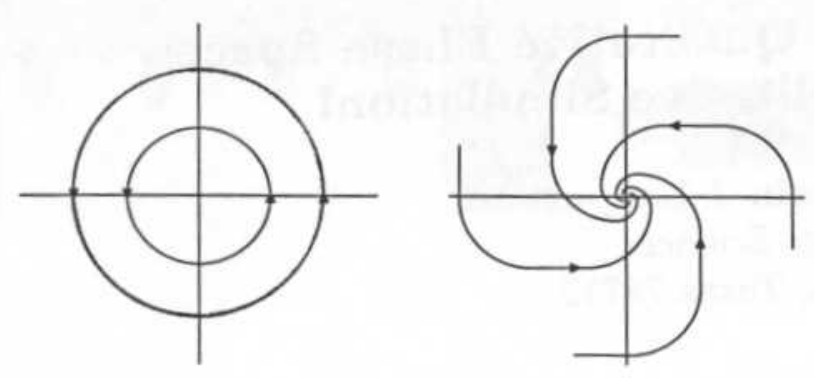

Figure 1: Some phase portrait of oscillatory systems.

\section{The Phase Space View}

The non-intersection constraint is based on the standard phase space representation for systems of first-order differential equations. An $n$th order equation can always be reduced to a system of $n$ first order equations. For example, the linear damped spring, described by the second order equation $m a=-k x-\eta v$, is also described by the following system of two first order equations:

$$
\begin{aligned}
\dot{x} & =v \\
\dot{v} & =-\frac{k}{m} x-\frac{\eta}{m} v
\end{aligned}
$$

A phase space for a system is the Cartesian product of a set of independent variables (state variables) that fully describes the system. For second order systems, this corresponds to a phase plane. A point in the phase space (phase point) represents a state of the system. Changes of the system over time define a trajectory through the phase space which tracks the state changes. Thus a trajectory is a geometrical representation of a solution to a system. A phase portrait (or phase diagram) for a system depicts its phase space and trajectories and is a geometrical representation of the qualitative behavior of the system. Figure 1 shows some phase portraits of oscillatory systems. From left to right, they represent solutions of steady oscillations and diminishing oscillations, respectively. For a more thorough treatment of the phase space representation, please refer to an elementary differential equations book such as [Boyce and diPrima, 1977].

A QSIM prediction is a qualitative description of the behavior of a solution to a given system (Figure 2). Thus it also describes the class of trajectories in the phase space which has the corresponding qualitative description. Using the Cartesian product of the quantity spaces of the state variables as the qualitative phase space, the trajectory of a QSIM prediction may be obtained by plotting the qualitative states predicted in this qualitative phase space.

\section{The Non-Intersection Constraint}

The mathematical foundation for the non-intersection constraint is a theorem about trajectories of autonomous systems which states that:

A trajectory which passes through at least one point that is not a critical point cannot cross itself

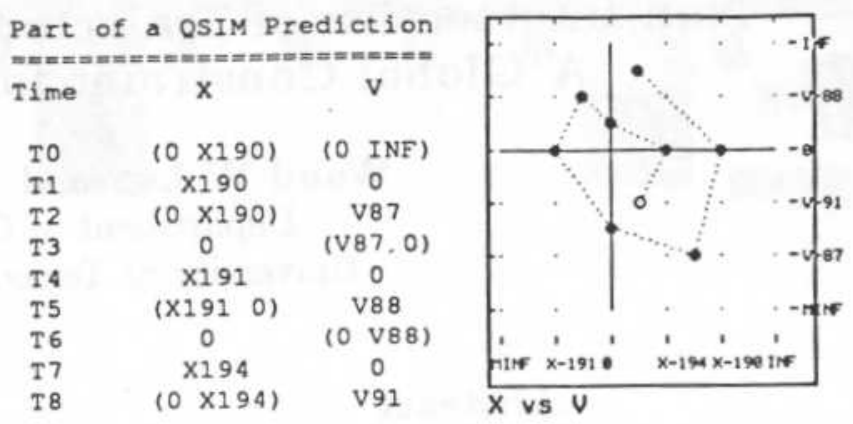

Figure 2: A QSIM prediction and its qualitative phase portrait.

unless it is a closed curve. In this case the trajectory corresponds to a periodic solution of the system [Boyce and diPrima, 1977, p.379-380].

Its proof follows from the existence and uniqueness theorems for systems of first order differential equations and will not be given here.

Autonomous systems are systems whose phase space representations do not explicitly involve the independent variable (time, in QSIM). Since QSIM deals with systems that do not involve explicit time functions, this theorem applies to the QSIM domain. The idea of the nonintersection constraint, then, is to implement the constraint imposed by this theorem onto trajectories of QSIM predictions.

The difficulty with applying this constraint within QSIM is that the qualitative description of behaviors only specifies values in terms of a discrete set of symbols, i.e. landmark values and the intervals between them. Therefore, we only know where the phase space trajectory is in a loose, qualitative sense. For example, in Figure 2, the precise trajectory from $(X 190,0)$ to $(X 191,0)$ is unknown. We only know that it reaches $V 87$ before crossing the negative $v$ axis.

If a trajectory consists of a single critical point, it will be a quiescent initial state and we need not worry about constraining its simulation. If on the other hand the trajectory is a closed curve, it corresponds to cyclic behavior and an appropriate filter in QSIM takes care of the behavior. Thus, we need only concern ourselves with multi-state, non-cyclic behaviors.

Given this, the problem then is to detect intersections between segments of a trajectory. The simplest case occurs when a trajectory reaches a point (coordinates specified by a pair of landmark values) it passed through before. In the general case, however, the intersection point lies between landmark values. We prove its existence for second order systems by establishing a criterion for intersection as described below.

Pick a trajectory segment with end points defining a rectangle which encloses all points of the segment. Consider segment $a c$ enclosed in rectangle $a b c d$ (Figure $3 \mathrm{a}$ ). The segment partitions the edges of the rectangle into two sets, $\{a b, b c\}$ and $\{a d, d c\}$. If the trajectory later enters this rectangle through one edges set, say $\{a b, b c\}$ at $b$, and 


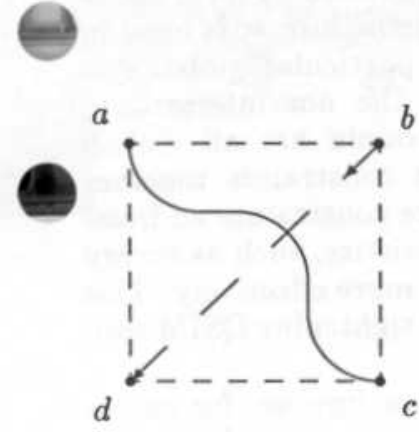

(a)

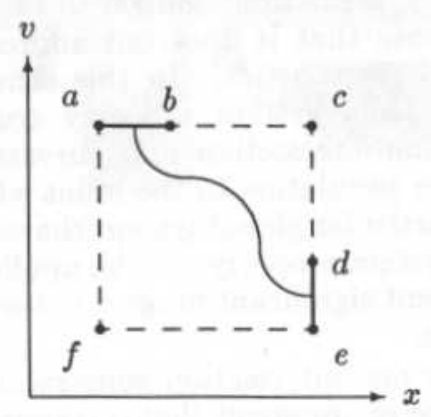

(b)
Figure 3: Intersection criterion for the non-intersection constraint.

exits through the other, in this case $\{a d, d c\}$ say at $d$, an intersection must occur, even if we don't know precisely where ${ }^{1}$. Establishing this condition for a trajectory is thus a criterion to conclude that the trajectory intersects itself. It is general and applies to all second order systems QSIM deals with.

\section{Implementation}

The non-intersection constraint has been implemented using the criterion for intersection just described. An interesting source of complication is that phase 'points' can be points, intervals or areas depending on whether the state variables are at landmarks or in intervals. Consider the case of Figure 3b. The state variable $x$ is in an interval

one end of a trajectory segment and at a landmark at we other end, and vice versa for the variable $v$. In this case, the edge sets satisfying the intersection criterion are $\{a f, f e\}$ and $\{b c, c d\}$, rather than $\{a f, f e\}$ and $\{a c, c e\}$. Other sources of complication are discussed in [Lee and Kuipers, 1988].

The non-intersection constraint is applied to all legitimate phase spaces of a system. This means that for the damped spring, the constraint is applied to each of the $x$ $v, v-a$ and $a-x$ phase spaces ${ }^{2}$. This is necessary because of the local point of view of limit-analysis-based qualitative simulation methods. Simply applying the constraint to, say, the $x-v$ space would not ensure that the parameter $a$ behaves properly.

\section{An Example}

We have chosen the damped spring as an example to illustrate the power of this constraint. The reason is that the damped spring is a representative second order system

\footnotetext{
${ }^{1}$ This is a direct consequence of the Jordan Curve Theorem which says that a closed curve in a plane divides the plane into exactly two regions. Refer to [Christenson and Voxman, 1977] for details.

${ }^{2}$ Normally, the $x-v$ space is considered the phase space for a damped spring. In fact, though, any collection of variables $t^{\prime} t$ is a linearly independent set and that fully describes the em can be the phase space.
}

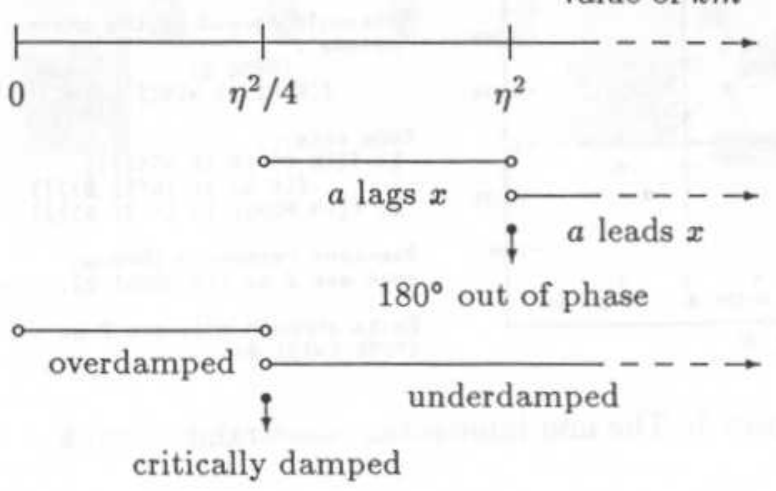

Figure 4: Correspondence between relative values of $\mathrm{km}$ and $\eta^{2}$ and behavior of linear damped spring.

with versions of varying complexity (from linear to nonlinear):

$$
\begin{array}{ll}
\text { linear damped spring } & m a=-k x-\eta v \\
\text { monotonic spring force } & m a=-f(x)-\eta v \\
\text { monotonic damping } \quad m a=-k x-g(v) \\
\text { general damped spring } \quad a=-f(x)-g(v)
\end{array}
$$

These same equations also describe damped oscillatory systems in other domains (e.g. circuits and control).

Damped spring systems have two types of behaviors, purely oscillatory and reaching quiescence. The division between these two types is, in the linear case, governed by the relationship between $4 \mathrm{~km}$ and $\eta^{2}$ (Figure 4 ). Its behavior is purely oscillatory (underdamped) if $4 \mathrm{~km}>\eta^{2}$ and reaches quiescence otherwise (overdamped and critically damped). For purely oscillatory behaviors, different phase relationships between $x$ and $a$ are possible and are, in the linear case, governed by the relationship between $\mathrm{km}$ and $\eta^{2}$.

Using the non-intersection constraint together with a curvature constraint [Kuipers and Chiu, 1987] on the damped spring systems has made predictions tractable. Three sets of behaviors are predicted. One set consists of strictly expanding oscillations with varying phase relationship between $a$ and $x$. Another consists of strictly diminishing oscillations with varying phase relationship between $a$ and $x$. The third consists of behaviors reaching quiescence after arbitrary number of diminishing oscillations.

Among these three sets, the expanding set is eliminated when energy constraints are included [Lee et al., 1987]. The system property constraints impose consistent $x$ - $a$ phase relationships on the remaining two sets. Since behaviors with overdamped and critically damped approaches to quiescence correspond to $4 \mathrm{~km} \leq \eta^{2}$, filtering the behaviors in the third set requires imposing constraints of a numerical nature. The quantitative reasoning methods of Kuipers and Berleant [1988] should make it possible to apply partial quantitative knowledge to filter these behaviors.

The behaviors of the damped spring system that survive the combined curvature, non-intersection, energy and 


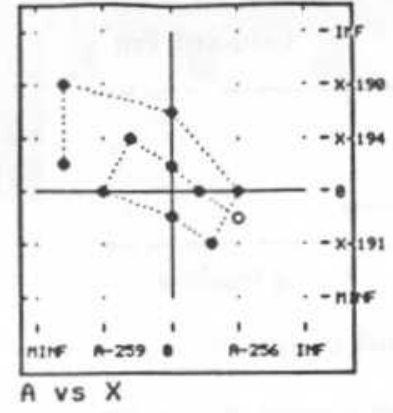

Intersection in $\mathrm{A}-\mathrm{X}$ portrait.

Rectangle forned by the phase 'points': [A256 B] [(8 A256) K191]

Edge sets:

1. $([(X$ B) (A $(B$ A256) ) ]

$[(A$ B) $(X(X 191$ B $))]]$

2. $([(A-A 256)(X(X 191$ B $))])$

Reenters rectangle through

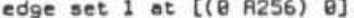

Exits through edge set 2 ot [A256 (X191 B)].

Figure 5: The non-intersection constraint at work.

system-property constraints can be classified as follows:

1. Overdamped or critically damped approach to quiescence.

2. Diminishing oscillations, with one of three constant $x-a$ phase relations.

3 . Diminishing oscillations, with varying $x-a$ phase relations.

4. Diminishing oscillations, reaching quiescence after an arbitrary finite number of oscillations.

All behaviors can be accounted for for each version of the damped spring. For the general damped spring and the monotonic damping cases, behaviors from all four classes are possible. For the monotonic spring force and linear cases, behaviors from classes 1,2 and 4 are predicted. However, only classes 1 and 2 represent possible behaviors in the linear case. Spurious predictions are due to limitations on the current form of the system property constraint. Incorporating Kuipers and Berleant's [1988] quantitative reasoning methods should allow us to eliminate them. Output showing the non-intersection constraint at work is included in Figure 5.

\section{Discussion}

Although the $\mathrm{M}^{+}$functional relationship is defined to be time invariant in QSIM, insufficient mechanisms are incorporated to ensure that QSIM treats each $M^{+}$function consistently. This is the reason why Lee et al. [1987] had limited success with nonlinear versions of the damped spring. For nonlinear versions of the damped spring, the envelopes derived for $a$ from the corresponding energy equations are too weak to constrain $a$ appropriately. Thus QSIM predicts that $a$ can behave more or less arbitrarily. This, however, gives rise to behaviors with inconsistent $M^{+}$functions which violate the non-intersection constraint. Applying the non-intersection constraint eliminates these spurious predictions.

In comparison with previously introduced constraints curvature, energy (Lyapunov) and system property, the non-intersection constraint is not system-specific in that its derivation does not depend on the particular system QSIM works on. Its form remains the same and it applies equally regardless of the system. The curvature constraint is fundamental in the sense that it addresses QSIM's lack of higher derivative information for performing local stateto-state predictions central to the algorithm. It is local in the sense that it does not address particular global system characteristics. In this sense, the non-intersection, energy and system property constraints are all global. The non-intersection and curvature constraints together tighten simulation to the point where constraints addressing particular global system characteristics, such as energy and system property, can be applied more effectively. This represent significant progess towards tightening QSIM simulation.

The non-intersection constraint can impose, for example, the requirement that a trajectory must spiral inwards, but it does not guarantee that the spiral converges to the origin. It remains possible that the spiral converges to a limit cycle. This ambiguity can be resolved using an appropriately chosen Lyapunov (energy) function.

Another possible approach for resolving this ambiguity is to apply aggregation methods [Weld, 1986] to abstract the decreasing oscillation to an amplitude decreasing towards zero. This abstraction transforms the ambiguity between asymptotically stable behavior and limit cycle to a much simpler limit-analysis type ambiguity. We need only ask whether a changing value (the amplitude) moving towards a limit (zero) reaches it or stops before reaching it.

In the current paper, we have discussed only the nonintersection constraint applied between two segments of the same trajectory. In fact, the non-intersection constraint applies more generally, prohibiting intersections between any two trajectories in the same phase portrait. This last condition raises an important subtlety. Two trajectories within the same phase portrait represent different possible initial conditions of the same system. However, since a set of QSIM predictions may have different presuppositions about the system properties of the system being simulated, it is not guaranteed that two arbitrarily chosen QSIM behaviors may be legitimately placed into the same phase portrait. Thus, in order to apply the nonintersection constraint between two trajectories, we must be able to determine whether their presuppositions about system properties are compatible. We plan to address this problem in future work.

\section{Related Work}

Struss [1987] has made a significant contribution to the mathematical foundations of qualitative reasoning through a careful analysis of qualitative algebras in terms of interval algebras. Kuipers [1988] elaborates on some of Struss' points, and clarifies a misconception about QSIM. In his appendix, Struss makes an interesting analysis of the spring without friction (the simple spring) based on the phase space approach. Using purely qualitative arguments (symmetry) about trajectories of the simple spring, he arrives at the conclusion that the simple spring oscillates with constant amplitude. He then adds that this would make adding further equations like conservation of energy unnecessary.

A point to note, however, is that the conservation of energy equation is not a further equation that needs to be added. It is derivable from the original description of the system. The process of deriving it would be liken to the 
process of his analysis. The difference is that knowledge A. algebraic manipulation is needed rather than of phase -pace trajectory analysis.

Sacks' work [1987] is impressive in automating the mathematician's analysis of precisely specified ODEs. Using a mbination of numerical and analytical methods (notably ecewise linear approximations), his PLR program produce qualitative descriptions of solutions, in the form of phase diagrams, for nonlinear differential equations. His approach is to first make a simple piecewise linear approximation of the given equations and construct phase diagrams for them. Then he refines his approximation, constructs another set of diagrams and compares them with the previous ones to look for new qualitative properties. This process of refine-and-compare continues until no new properties are found. His program performs well on a variety of equations.

Our work addresses the problem of obtaining qualitative behaviors from an incompletely specified QDE. When key functional relations are known only to lie in the class of monotonic functions, piecewise linear approximation is impossible, and Sacks' powerful methods do not apply.

\section{Conclusions}

QSIM is a powerful inference mechanism for predicting qualitative solutions of QDEs. However, if unconstrained, it is possible for QSIM to predict intractable spurious solutions.

Kuipers and Chiu [1987] and Lee et al. [1987] have introduced various constraints to tighten the simulation process. They are useful, but are in general unable to tighten simulation to the point where predictions become tractable.

We have introduced a global, non-system-specific con-

aint to eliminate a major source of spurious predictions.

is is the non-intersection constraint for phase space trajectories which specifies that a trajectory cannot intersect itself. Using it and the curvature constraint together tightens simulation to the point where other global and systemspecific constraints can be applied more effectively. This is demonstrated on damped oscillatory systems.

Introduction of the non-intersection constraint represents significant progress towards tightening QSIM simulation. Current implementation applies the constraint between two segment of the same trajectory. Future work includes generalizing the constraint to apply between trajectroies and automating interpretation of behavior classes, for example, by aggregation of repeated cycles [Weld, 1986], or by merging behaviors into families [Chiu, 1988].

\section{Acknowledgments}

Thanks to Charles Chiu, Xiang-Seng Lee, Jason See and Wing Wong for reading drafts of this paper.

\section{References}

[Boyce and diPrima, 1977] W. E. Boyce and R. C. DiPrima. Elementary Differential Equations. John Wiley \& Sons, New York, 1977.

[Chiu, 1988] C. Chiu. Automatic Analysis of Qualitative Simulation Models. Unpublished, 1988.

[Christenson and Voxman, 1977] C. O. Christenson and W. L. Voxman. Aspects of Topology. Marcel Dekker, New York, 1977.

[Kuipers, 1986] B. J. Kuipers. Qualitative Simulation. Artificial Intelligence 29: 289-338, 1986.

[Kuipers and Chiu, 1987] B. J. Kuipers and C. Chiu. Taming Intractable Branching in Qualitative Simualtion. IJCAI-87, 1987.

[Kuipers, 1988] B. J. Kuipers. The Qualitative Calculus is Sound but Incomplete: A Reply To Peter Struss. To appear in International Journal of $A I$ in Engineering, 1988.

[Kuipers and Berleant, 1988] B. J. Kuipers and D. Berleant. Using Incomplete Quantitative Knowledge in Qualitative Reasoning. AAAI-88, 1988.

[Lee et al., 1987] W. W. Lee, C. Chiu and B. J. Kuipers. Developments Towards Constraining Qualitative Simulation. UT TR AI87-44. Also in AAAI-87 Qualitative Physics Workshop Abstracts, 1987.

[Lee and Kuipers, 1988] W. W. Lee and B. J. Kuipers. Non-Intersection of Trajectories in Qualitative Phase Space: A Global Constraint for Qualitative Simulation. TR forthcoming. 1988 .

[Sacks, 1987] E. P. Sacks. Piecewise Linear Reasoning. AAAI-87, 1987.

[Struss, 1987] P. Struss. Problems of Interval-Based Qualitative Reasoning. Siemens Corp., ZTIINF, 1987.

[Weld, 1986] D. S. Weld. The Use of Aggregation in Causal Simulation. Artificial Intelligence 30: 1-34, 1986. 